\title{
Applications and challenges of artificial intelligence in diagnostic and interventional radiology
}

\author{
Joseph Waller ${ }^{1, A, D, E}$, Aisling $0^{\prime} C_{0 n n o r}^{2, B, E, F}$, Eleeza Raafat ${ }^{3, B, D, E, F}$, Ahmad Amireh ${ }^{4, E, F}$, John Dempsey, ${ }^{5, E, F}$, \\ Clarissa Martin ${ }^{6, E, F}$, Muhammad Umair ${ }^{7,0, E}$ \\ 'Drexel University College of Medicine, USA \\ 2University of Washington, Seattle, WA 98195, USA \\ ${ }^{3}$ Loyola University Chicago Stritch School of Medicine, Maywood, IL 60153, USA \\ ${ }^{4}$ Duke University, Department of Biology, Durham, NC 27708, USA \\ ${ }^{5}$ Boston College, Chestnut Hill, MA 02467, USA \\ ${ }^{6}$ University of Pennsylvania, Philadelphia PA 17101, USA \\ ${ }^{7}$ Northwestern University Feinberg School of Medicine, Department of Radiology, Chicago, IL, USA
}

\section{Abstract}

Purpose: Machine learning (ML) and deep learning (DL) can be utilized in radiology to help diagnosis and for predicting management and outcomes based on certain image findings. DL utilizes convolutional neural networks $(\mathrm{CNN})$ and may be used to classify imaging features. The objective of this literature review is to summarize recent publications highlighting the key ways in which ML and DL may be applied in radiology, along with solutions to the problems that this implementation may face.

Material and methods: Twenty-one publications were selected from the primary literature through a PubMed search. The articles included in our review studied a range of applications of artificial intelligence in radiology.

Results: The implementation of artificial intelligence in diagnostic and interventional radiology may improve image analysis, aid in diagnosis, as well as suggest appropriate interventions, clinical predictive modelling, and trainee education. Potential challenges include ethical concerns and the need for appropriate datasets with accurate labels and large sample sizes to train from. Additionally, the training data should be representative of the population to which the future ML platform will be applicable. Finally, machines do not disclose a statistical rationale when expounding on the task purpose, making them difficult to apply in medical imaging.

Conclusions: As radiologists report increased workload, utilization of artificial intelligence may provide improved outcomes in medical imaging by assisting, rather than guiding or replacing, radiologists. Further research should be done on the risks of $\mathrm{AI}$ implementation and how to most accurately validate the results.

Key words: artificial intelligence, machine learning, radiology, imaging.

\section{Introduction}

Artificial intelligence (AI) and machine learning (ML) are umbrella terms for computational models that can learn from data and encompass the evolution of artificial neu- ral networks (ANN) and deep learning (DL). An ANN is a linkage of multiple layers of 'neurons', and if one or more of those layers involves a convolutional filter, then the network is defined as a convolutional neural network (CNN). $\mathrm{DL}$ involves training $\mathrm{CNN}$ and includes an input layer

Correspondence address:

Joseph Waller, Drexel University College of Medicine, USA, e-mail: jw3642@drexel.edu

Authors' contribution:

A Study design · B Data collection · C Statistical analysis · D Data interpretation · E Manuscript preparation · F Literature search · G Funds collection 
that feeds its output to the first hidden layer, followed by the second, and eventually to the output layer (each layer consists of nodes loosely modelled from neurons).

Both ML and DL require training periods, of which there are 2 different types: supervised and unsupervised. Supervised learning utilizes labelled data and thus is used for classification and regression, whereas unsupervised learning uses unlabelled data and thus can only recognize patterns through clustering and dimensionality reduction. ML may be used in radiology by identifying specific conditions or partitioning images into parts. Some of the ways in which ML has been used include detecting fatty liver via ultrasound (US), characterizing carotid plaque via computed tomography $(\mathrm{CT})$, and predicting lesion-specific ischaemia via quantitative coronary CT angiography $[1,2]$. There are several advantages and disadvantages of applying ML in this context. ML can process huge amounts of data and identify trends and patterns that may otherwise be undetectable. However, extensive highquality datasets are required for adequate training.

DL, however, allows for more complex classification as well as automatic feature extraction and learning. Hybrid strategies that involve some degree of human contribution are often used. ANN systems were historically limited by a lack of computing power and training data. However, considering the relatively recent technological and mathematical advances, in addition to the availability of big data, ANN systems are being re-evaluated for their application in medical imaging [1]. Radiologists have historically been at the forefront of technology in medicine and are in a prime position to guide the incorporation of AI into medicine [2]. Given the increased workload, it is predicted that incorporating $\mathrm{AI}$ in radiology would assist rather than guide or replace radiologists, allowing them a more central role in patient care due to its diagnostic support via image classification and outcome/risk predictions $[3,4]$.

This literature review will provide a historical context to utilizing artificial intelligence in medical imaging, highlight ways in which artificial intelligence may be applied in interventional radiology, and acknowledge the challenges that physicians may face during this implementation. It will also provide a variety of suggestions for overcoming these problems.

\section{Material and methods}

Articles published between 1 January 2017 and 1 December 2020 were searched on the PubMed database. Keywords including "Artificial Intelligence" OR "Machine Learning" AND "Radiology" were utilized in our search of the abstract and/or titles of the articles. Studies on animal models were excluded, as well as studies with redundant information and overlapping patient cohorts. Beyond that, the authors screened the abstracts for articles with findings relevant to our topic. Ultimately, 21 studies that provided the most relevant information were included in this literature review, some of which are highlighted in Table 1.

\section{Results}

\section{Applications}

Implementing AI in DR and IR may improve image analysis, directly increasing the efficacy of image analysis and efficiency of workflow as seen in the study by Do et al., in which clinicians were notified sooner by a median of 1 hour and radiologist exam interpretation time was decreased by $37 \%$ [5]. Similarly increased efficiency and efficacy using AI were demonstrated by Gore [6]. Potential applications involving image analysis are broad, including but not limited to imaging in pulmonary, rheumatology, and cardiology domains in addition to optimization of dose distributions for imaging modalities using ionizing radiation. For example, a reduction of unnecessary radiation to non-target organs by utilization of AI has been described [7-10]. Angiography-based ML allows real-time fractional flow reserve estimates, which may be expanded to estimating physiological data from fluoroscopic images [9]. Automatic vessel analysis is already utilized as this technology paves the way to analysing vessel size, lesion characteristics, and post-treatment effects. Additionally, a virtual CT image can be created from MRI data, which may lead to combining pre-procedural 3D images onto intraoperative 2D images by syncing MRI or CT imaging with intraoperative fluoroscopic or US images [9]. Advances in image analysis stand to help developing countries that have less access to diagnostic tests/medical care by improving tuberculosis diagnosis through chest radiography, CADs, and DL algorithms, which is especially appealing given that tuberculosis is a leading cause of death from infectious disease worldwide [11].

Within the field of IR, AI also shows promise in clinical predictive modelling. A current difficulty in IR is estimating the benefit of a treatment prior to its completion [12]. Accurately doing so would minimize risk to the patient, diminish healthcare costs, and reduce unnecessary treatments [12].The application of AI, specifically DL, is promising in this setting as a prediction model that would improve itself with increased use and feedback [12]. $\mathrm{AI}$ is used in hepatocellular carcinoma staging systems by incorporating data on responders vs. non-responders to various treatments [13]. The adoption of DL could mitigate a major challenge of IR, which is estimating the outcomes/benefits of a treatment prior to performing it [12]. In addition, there are other ways in which AI may prove to be an asset to physicians. For example, AI also holds potential in touchless procedural guidance and support (e.g. eye-tracking, inertial sensors, cameras/ 
Table 1. Applications and Challenges of Al in radiology

\begin{tabular}{|c|c|c|}
\hline Study & Applications & Challenges \\
\hline Lee et al. [1] & $\begin{array}{l}\text { Image segmentation and registration } \\
\text { Automatic labelling and captioning } \\
\text { CAD }\end{array}$ & $\begin{array}{l}\text { Quality and amount of training data } \\
\text { Explaining "technical bases" of the system } \\
\text { Legal/ethical issues }\end{array}$ \\
\hline Sailer et al. [4] & Diagnostic support through classification of images and outcome/risk predictions & N/A \\
\hline Do et al. [5] & $\begin{array}{l}\text { Automation using Al notified clinicians faster by a median of } 1 \text { hour and decreased } \\
\text { radiologist exam interpretation time by } 37 \% \\
\text { Concordance of target lesion measurements improved from } 22.5 \% \text { to } 67.8 \%\end{array}$ & N/A \\
\hline Chassagnon et al. [7] & $\begin{array}{l}\text { Thoracic imaging, specifically lung nodule evaluation, tuberculosis/pneumonia } \\
\text { detection, and quantification of diffuse lung diseases }\end{array}$ & $\begin{array}{l}\text { Current algorithms are limited to } \\
\text { isolated findings }\end{array}$ \\
\hline Stoel et al. [8] & Rheumatological imaging with a focus on rheumatoid arthritis and systemic sclerosis & N/A \\
\hline Maurowski et al. [9] & $\begin{array}{l}\text { Improve disease detection, decrease unnecessary procedures, improve outcomes, } \\
\text { and reduce costs }\end{array}$ & $\begin{array}{l}\text { Concern for diminished pay and prestige } \\
\text { for radiologists } \\
\text { Goals of Al developers may not coincide } \\
\text { with the altruistic goals of healthcare }\end{array}$ \\
\hline Poortmans et al. [10] & Dose distribution optimization to reduce unnecessary radiation to non-target organs & N/A \\
\hline Kulkarni et al. [11] & $\begin{array}{l}\text { Tuberculosis diagnosis through chest radiography, computer-aided diagnosis } \\
\text { systems, and DL algorithms }\end{array}$ & N/A \\
\hline lezzi et al. [12] & $\begin{array}{l}\text { Pattern recognition and identification, language comprehension, object and } \\
\text { sound recognition, prognosticating diseases, determining indication for therapy, } \\
\text { estimating the outcomes/benefits }\end{array}$ & $\begin{array}{l}\text { High quality data sets are required } \\
\text { for training } \\
\text { Often do not disclose the statistical } \\
\text { rationale, which makes medical } \\
\text { application difficult }\end{array}$ \\
\hline Meek et al. [13] & $\begin{array}{l}\text { Imaging, prediction modelling, and decision support } \\
\text { Angiography-based ML to provide real-time estimates of fractional flow reserve, } \\
\text { which is used to identify ischaemia-related stenosis in coronary artery disease } \\
\text { Fusion of images would allow precise guidance during procedures } \\
\text { Generate CT image from MRI data } \\
\text { ML algorithms may be used to guide treatment plans } \\
\text { Combining ML with augmented reality systems offers a new method of training } \\
\text { and testing trainees }\end{array}$ & $\begin{array}{l}\text { Large amounts of data would be } \\
\text { required to train the algorithms, } \\
\text { which is further complicated by } \\
\text { the ever-changing nature of clinical } \\
\text { practice, which may limit"the usefulness } \\
\text { of retrospective data" }\end{array}$ \\
\hline
\end{tabular}

webcams, and voice-driven assistants), which currently must be prepared prior to a procedure, largely due to sterility concerns, but would be helpful to access during the procedure [12]. The collaboration between computer scientists, biomedical engineers, and interventional radiologists would pave the way to superior patient care in the near future.

The inclusion of AI into IR may not be limited to clinical practice, but also in educating future or training clinicians. If AI were to be combined with augmented reality (AR) systems, this would present a new method of training and testing trainees [13]. There already exists an orthopaedic-specific surgical simulation using patient-specific anatomic modelling data acquired by cross-sectional imaging and manual image segmentation, but incorporating ML into the process would improve this process and thus allow for more procedural simulations to be developed [13]. In the future, similar simulations designed for interventional radiology trainees may help to develope trainees' education and skill.

\section{Challenges}

One potential challenge to the implementation of AI in IR involves ethics, specifically involving conflicts of interest of radiologists and AI developers. It is important for radiologists and AI researchers to be mindful of their biases and personal motivations and to focus on alleviating the suffering of patients by providing the best care and technology $[9,13]$. Radiologists may be wary of diminished employment opportunities, prestige, and pay. However, radiologists have a moral obligation to advocate for research and implementation of that which best supports patient care. Furthermore, radiologists are reporting increased workload [2] and notably, it is emphasized that incorporating AI in radiology would assist rather than guide or replace radiologists, allowing them a central role in patient care due to its diagnostic support via image classification and outcome/risk predictions $[3,4]$. This implies that implementing AI would be largely beneficial for radiologists. There are also ethical concerns in- 
volving AI developers, specifically that they may not share the altruistic goals of healthcare and thus may avoid sharing data between companies, for example [9]. However, sharing data is vital to developing robust AI because large amounts of data are required for training. It is also important that developers avoid seeking excessive attention/ funding for their discoveries and mind the limitations of the technology. A joint European and North American multi-society [14] came to a consensus that AI may introduce highly consequential systemic errors and that it is not yet clear how to best integrate $\mathrm{AI}$ into clinical practice [14]. More research is called for to mitigate such potential systematic errors; however, justified use of this technology would promote well-being, minimize harm, and ensure benefits/harm are distributed among stakeholders justly [14]. Freedom, dignity, privacy, maximum transparency, dependability, responsibility, accountability, and diversity and inclusion must all play a role in the code of ethics and practice for any $\mathrm{AI}$ applications intended to be used in medicine [14]. This code should emphasize helping patients and the common good, bar the use solely for financial gain, and act as a step towards mitigating the ethicsrelated challenges presented [14].

Another potential challenge posed by AI is the substantial number of data required to train the algorithms. Through clinical research and technological advancements, a large number of data have been produced [15]. AI would be a good option to bridge the gap between the complex data obtained thus far and clinical decision making, allowing proper utilization of the collected data. However, this is further complicated by the ever-changing nature of clinical practice, which may limit the pool of relevant retrospective data [13]. Additionally, given that the amount of data utilized to train the ML system dictates the confidence in the power of the prediction or judgement of the output, there may be inherent inaccuracies for the application of such algorithms among groups who are under-represented in the population under test.

Clinical implementation of AI may be limited in other ways as well. Machines often do not disclose the statistical rationale behind the elaboration of their tasks, which makes it complicated to apply in a medical setting [12]. This means that it is impossible to understand the rationale behind the final output of a DL algorithm as to why a particular output is assigned, especially in cases where the output does not conform with the opinion of an expert radiologist or when the output produces a result that turns out to be incorrect when correlated to a gold-standard test. In such cases, there is no rational basis identified to justify the results.

Additionally, AI is limited by interpretability and the large number of annotated data required [16]. Combining multiple technologies with human experts yields the most accurate results, and it is suggested that AI be used in conjunction with randomized controlled trials to provide insight into causality $[12,17]$. While some view AI as aimed to improve current automation technologies, others are hopeful that AI will help address low-value utilization, higher cost of imaging, and high work volume for radiologists interpreting images $[16,18,19]$.

\section{Discussion}

It is predicted that AI will contribute significantly to medical imaging over the next 5-10 years [20]. CT images are already constructed using various reconstruction algorithms to generate a final image to be displayed. Additionally, many image analysis tools have existed in practice for a long time that have utilized some sort of artificial intelligence. However, with the advancement of the field and widespread use of deep neural networks, there have been breakthroughs for medical imaging. For example, CT reconstructions may be improved by GAN (generative adversarial network; an ML model that generates its own training data) and radiomics/DL-based image analysis. Currently, AI is being studied for almost every imaging modality and in every subspecialty of medical imaging for most pathologies in a multitude of research settings. However, additional research should also be done on the risks of using AI in image analysis and clinical predictive modelling as well as potential challenges and pitfalls with implementation of these technologies. There should also be more research into what constitutes adequate validation of work produced by AI.

Additionally, analysis of $38 \mathrm{AI}$ articles from earlymid 2018 revealed that standardized reporting of ML/ DL methods would address approximately one-third of reviewer critiques [21]. Standardized radiology reporting would certainly be an important consideration going forward as AI is researched and implemented in IR, because it provides a well-established framework to import useful information from radiology reports to generate labels used to train the AI.

\section{Limitations of the study}

One of the limitations of this review is its relatively small sample size of reviewed articles. Twenty-one sources were analysed, and while they were all published within the last 1-2 years, this review only focused on a short period of time. The ethical concerns raised are primarily supported by 2 sources: one is a joint multi-society statement and the other raised ethical concerns of conflict of interest between radiologists and AI developers $[9,14]$. This is a very small sample size with which to discuss current ethical concerns surrounding AI in IR.

\section{Conclusions}

This review evaluated 21 publications to determine the applications and challenges of AI in diagnostic and interventional radiology. Ethical concerns like ensuring 
the AI is trained with the appropriate patient population remain, as well as the need for research on how to most accurately validate results. Nevertheless, these papers have demonstrated AI's potential, which includes the ability to aid in diagnosis, suggest appropriate interventions, and especially to improve image analysis. In a time when radiologists are reporting increased workload, utilizing AI may be an answer to improving efficacy and efficiency, allowing more time for radiologists to spend on interpretation and other cognitive aspects of the job.

\section{Conflict of interest}

The authors declare no conflict of interest.

\section{References}

1. Lee JG, Jun S, Cho YW, et al. Deep learning in medical imaging: general overview. Korean J Radiol 2017; 18: 570-584.

2. Alexander A, Jiang A, Ferreira C, Zurkiya D. An intelligent future for medical imaging: a market outlook on artificial intelligence for medical imaging. J Am Coll Radiol 2020; 17 (1 Pt B): 165-170.

3. Mohan CSM. Artificial intelligence in radiology - are we treating the image or the patient? Indian J Radiol Imaging 2018; 28: 137-139.

4. Sailer AM, Tipaldi MA, Krokidis M. AI in interventional radiology: there is momentum for high-quality data registries. Cardiovasc Intervent Radiol 2019; 42: 1208-1209.

5. Do HM, Spear LG, Nikpanah M, et al. Augmented radiologist workflow improves report value and saves time: a potential model for implementation of artificial intelligence. Acad Radiol 2020; 27: 96-105.

6. Gore JC. Artificial intelligence in medical imaging. Magn Reson Imaging 2020; 68: A1-A4.

7. Chassagnon G, Vakalopoulou M, Paragios N, Revel MP. Artificial intelligence applications for thoracic im-aging. Eur J Radiol 2020; 123: 108774 .

8. Stoel B. Use of artificial intelligence in imaging in rheumatology current status and future perspectives. RMD Open 2020; 6: e001063. doi: 10.1136/rmdopen-2019-001063.

9. Mazurowski M. Artificial intelligence in radiology: some ethical considerations for radiologists and algo-rithm developers. Acad Radiol 2020; 27: 127-129.

10. Poortmans PMP, Takanen S, Marta GN, Meattini I, Kaidar-Person O. Winter is over: the use of artificial intelligence to individualise radiation therapy for breast cancer. Breast 2020; 49: 194-200.

11. Kulkarni S, Saurabh J. Artificial intelligence, radiology, and tuberculosis: a review. Acad Radiol 2020; 27: 71-75.
12. Iezzi R, Goldberg SN, Merlino B, Posa A, Valentini V, Manfredi R. Artificial intelligence in interventional radiology: a literature review and future perspectives. J Oncol 2019; 2019: 6153041.

13. Meek RD, Lungren MP, Gichoya JW. Machine learning for the interventional radiologist. AJR Am J Roentgenol 2019; 213: 782-784.

14. Geis JR, Brady AP, Wu CC, et al. Ethics of artificial intelligence in radiology: summary of the joint European and North American multisociety statement. Can Assoc Radiol J 2019; 70: 329-334.

15. Letzen B, Wang CJ, Chapiro J. The role of artificial intelligence in interventional oncology: a primer. J Vasc Interv Radiol 2019; 30: 38-41.e1. doi: 10.1016/j.jvir.2018.08.032.

16. Nensa F, Demircioglu A, Rischpler C. Artificial intelligence in nuclear medicine. J Nucl Med 2019; 60 Suppl 2: 29S-37S.

17. Patel BN, Rosenberg L, Willcox G, et al. Human-machine partnership with artificial intelligence for chest radiograph diagnosis. NPJ Digit Med 2019; 2: 111.

18. Jha S, Cook T. Artificial intelligence in radiology - the state of the future. Acad Radiol 2020; 27: 1-2. doi: 10.1016/j.acra.2019.11.003.

19. Driver CN, Bowles BS, Bartholmai BJ, Greenberg-Worisek AJ. Artificial intelligence in radiology: a call for thoughtful application. Clin Transl Sci 2020; 13: 216-218.

20. Haubold J. Artificial intelligence in radiology: what can be expected in the next few years? Radiologe 2020; 60: 64-69 [Article in German].

21. Gregory J, Welliver S, Chong J. Top 10 reviewer critiques of radiology artificial intelligence (AI) articles: qualitative thematic analysis of reviewer critiques of machine learning/deep learning manuscripts submitted to JMRI. J Magn Res Imaging 2020; 52: 248-254. 